\title{
Conformational flexibility of the pharmacologically important insulin analogues
}

\author{
Olga Ksenofontova, Vasily Stefanov \\ Department of Biochemistry, Saint Petersburg State University, Saint Petersburg, Russia \\ Email: jabiata@yandex.ru
}

Received 12 August 2013; revised 15 September 2013; accepted 21 September 2013

Copyright (C) 2013 Olga Ksenofontova, Vasily Stefanov. This is an open access article distributed under the Creative Commons Attribution License, which permits unrestricted use, distribution, and reproduction in any medium, provided the original work is properly cited.

\begin{abstract}
Understanding the conformational flexibility of the insulin drugs is of great importance for the treatment of diabetes mellitus. Once in the body, the drug must have a certain degree of mobility within a specified period of time for the manifestation of its pharmacological properties. This mobility ensures conformational states necessary for binding with the insulin receptor and activating specific biological processes. In this work we investigated conformational flexibility of the pharmacologically important insulin analogues-insulin lispro, insulin aspart, insulin glulisine, and insulin glargine, using the molecular dynamics simulation method. This study provides new insight into the nature of behaviour of $A$ - and B-chains. It has been found out that B-chain substitutions result in rapid acting, while long-lasting action can be achieved by substitutions in both chains. The results of this study can be used for development of new insulinbased antidiabetic drugs.
\end{abstract}

Keywords: Insulin Analogues; Conformational Flexibility; Molecular Dynamics Simulation Study

\section{INTRODUCTION}

The current estimates of the World Health Organization indicate that more than 360 million people world-wide are diabetic [1]. These estimates suggest that the world is facing an unprecedented epidemic of diabetes mellitus, chiefly due to changes in the lifestyle, nutritional and environmental factors. Diabetes mellitus is a chronic disease, which occurs due to inefficient production (type 1 diabetes) or improper utilization (type 2 diabetes) of a protein hormone known as insulin [2]. Insulin is a small globular protein containing two chains: A (21 residues) and $\mathrm{B}$ (30 residues) [3]. The A-chain contains an $\mathrm{N}$-ter- minal $\alpha$-helix, non-canonical turn, and the second helix; the B-chain contains an $\mathrm{N}$-terminal segment, central $\alpha$ helix, and $\mathrm{C}$-terminal $\beta$-strand. There are three disulfide bonds in insulin molecule: two bonds between A- and B-chains and one bond inside A-chain. In solution at neutral $\mathrm{pH}$ and at physiological concentrations (about 1 $\mathrm{ng} / \mathrm{ml}$ ) insulin exists as a monomer [4]. Monomer is an active form of insulin. At higher concentrations, acid or neutral $\mathrm{pH}$ (in the absence of zinc) insulin self-associates to form dimers and hexamers (in the presence of zinc) [5].

The hexamer-monomer conversion is one of the central aspects of insulin formulations for injection [6]. The hexamer is far more stable than the monomer, but only the monomer form is biologically active. It is known that for the daily keeping of the glucose balance, the human body needs two forms of insulin: bolus (needed after meals) and basal (supplies the basal level of insulin for the day) [7]. For these purposes two types of insulin analogues have been developed: those that are more readily absorbed from the injection site and therefore act faster than natural insulin injected subcutaneously (rapidacting insulin analogues); and those that are released slowly over a period of between 8 and 24 hours (longacting insulin analogues). The rapid-acting analogues are insulin lispro (Humalog), insulin aspart (NovoRapid), and insulin glulisine (Apidra) [8]. The long-acting analogues are insulin glargine (Lantus), and insulin detemir (Levemir) [9]. All these analogues, except for insulin detemir, have some pharmacologically important amino acid substitutions in the primary structure of insulin (Figure 1).

Insulin lispro has reverse penultimate lysine and proline residues on the C-terminus of the B-chain [10]. This modification does not alter insulin receptor binding, but blocks the formation of insulin dimers and hexamers. Insulin aspart has an insulin aspartic acid instead of proline at B28 [11]. This substitution prevents the formation of hexamers. The third rapid insulin analogue is 


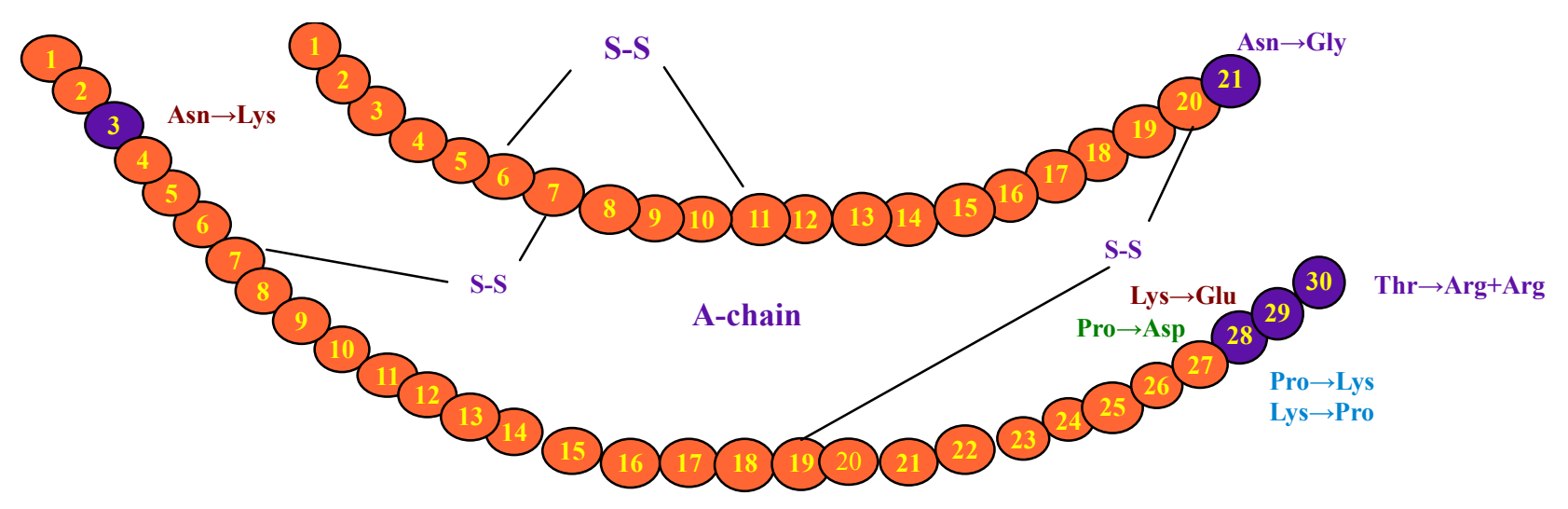

\section{B-chain}

Figure 1. Amino acid substitutions in insulin (orange balls—nonsubstituted sites; magenta balls—substituted sites) Substitutions: cyan-insulin lispro (B28, B29), green—insulin aspart (B28), red—insulin glulisine (B3, B29), magenta-insulin glargine (A21, addition to B30).

insulin glulisine $\left(\mathrm{Asn}^{\mathrm{B} 3} \rightarrow \mathrm{Lys}^{\mathrm{B} 3}, \mathrm{Lys}^{\mathrm{B} 29} \rightarrow \mathrm{Glu}^{\mathrm{B} 29}\right)$ [12]. Insulin glargine is a long-acting insulin analogue [13]. Two positively charged arginine were added to the Cterminus of the B-chain. They shift the isoelectric point from 5.4 to 6.7 , making this compound more soluble at a lightly acidic $\mathrm{pH}$ and less soluble at a physiological $\mathrm{pH}$. Replacing the acid-sensitive asparagine at A21 by glycine is used to avoid deamination and dimerization.

Traditionally, the behaviour of insulin analogues has been investigated using biochemical [14] and clinical methods [15]. However, they are rather rough and not able to explain mechanisms of interactions between insulin and the insulin receptor at the molecular level.

The molecular dynamics (MD) simulation method provides new insights concerning the internal motions of the proteins [16]. With continuing advances in the methodology and the speed of computers, MD studies are being extended to larger systems, greater conformaitonal changes and longer time scales [17]. This makes it possible to investigate motions and to obtain information that is not accessible from experiment.

The previous MD studies were focused on investigation of insulin conformational flexibility [18-21]. The aim of our study is to investigate the conformational flexibility of the pharmacologically important insulin analogues: insulin lispro, insulin aspart, insulin glulisine, and insulin glargine.

\section{MATERIALS AND METHODS}

All MD simulations were carried out using the GROMACS software package (version 4.5.5) [22]. The starting structure of the human insulin was obtained in Protein Data Bank (PDB code 1BEN). The homology models of insulin analogues (insulin lispro, insulin aspart, insulin glulisine, and insulin glargine) have been constructed using the MODELLER software (version 9.9) [23]. Obtained with the MODELLER molecules, were immersed in solvent using the tip4p water model. The solvated proteins were placed in the cubic boxes with periodic conditions and the system's energy was minimized to remove steric clashes.

To compute the forces acting on each atom in reasonable time, OPLS-AA (all-atom) force field was applied [24]. It approximates the potential energy of each atom, using different terms to bonded and non-bonded terms as follows (1):

$$
U=E_{\text {bonded }}+E_{\text {non-bonded }}
$$

The bonded term can be split up into three terms (2):

$$
E_{\text {bonded }}=E_{\text {bond-stretch }}+E_{\text {angle-bend }}+E_{\text {rab }}
$$

( $E_{\text {rab }}$ equals $E_{\text {rotate-along-bonds }}$ )

All three terms can be configured using a parameter $k$. The first term is a harmonic potential representing the length displacement from their ideal value $b_{0}$ of atomic pairs that are covalently bound (3):

$$
E_{\text {bond-stretch }}=\sum_{\text {covalent-bonds }} k_{b}\left(b-b_{0}\right)^{2}
$$

The second term accounts for the alteration of bond angles $\theta$ from ideal values $\theta_{0}$ and is also represented by a harmonic potential (4):

$$
E_{\text {angle-bend }}=\sum_{\text {angles }} k_{\theta}\left(\theta-\theta_{0}\right)^{2}
$$

Lastly, the third term is assumed to be periodic and therefore often expressed by a cosine function and accounts for twisting a bond (5): 


$$
E_{\text {rotate-along-bond }}=\sum_{\text {torsions }} k_{\varphi}(1-\cos (n \varphi))
$$

The non-bonded term is the sum of van-der-Waals and electrostatic interaction (6):

$$
E_{\text {non-bonded }}=E_{\text {van-der-Waals }}+E_{\text {electrostatic }}
$$

The NVT (constant number of particles, constant volume, and constant temperature) thermodynamic ensemble, followed by NPT (constant pressure) ensemble, was simulated during the 100 ps equilibration of the system. The temperature was held at $300 \mathrm{~K}$ using the V-rescale thermostat, and pressure using Parinello-Rahman barostat. Non-bonded interactions were cut-off beyond $10 \AA$. Long-range electrostatic interactions were handled using the particle mesh Ewald (PME) method with a $1 \AA$ grid spacing. MD simulations were conducted during 10 and $100 \mathrm{~ns}(10,000$ and 100,000 ps). Visualization and analysis of the conformation flexibility were performed using the VMD software (version 1.9) [25].

\section{RESULTS}

\subsection{Root Mean Square Deviations Analysis}

The protein stability during the simulations was assessed by calculating the root mean square deviations (RMSD). Figure 2 presents the RMSD evolution of backbone atoms with reference to the crystal structures of insulin aspart, insulin glulisine, insulin lispro, and normal insulin.

These curves show that the molecules are generally well-equilibrated at about 8000 ps ( $8 \mathrm{~ns}$ ). The most conformationally flexible rapid-acting analogue is insulin aspart. The equilibration of insulin aspart and the normal insulin begins at 5000 ps, insulin glulisine - at 3000 ps, and insulin lispro-at 7000 ps. We suggest that the equilibration time of these analogues is different because of chemical modifications at B28 (Figure 1).

Figure 3 presents the RMSD evolution of backbone atoms for all insulin analogues, including insulin glargine.

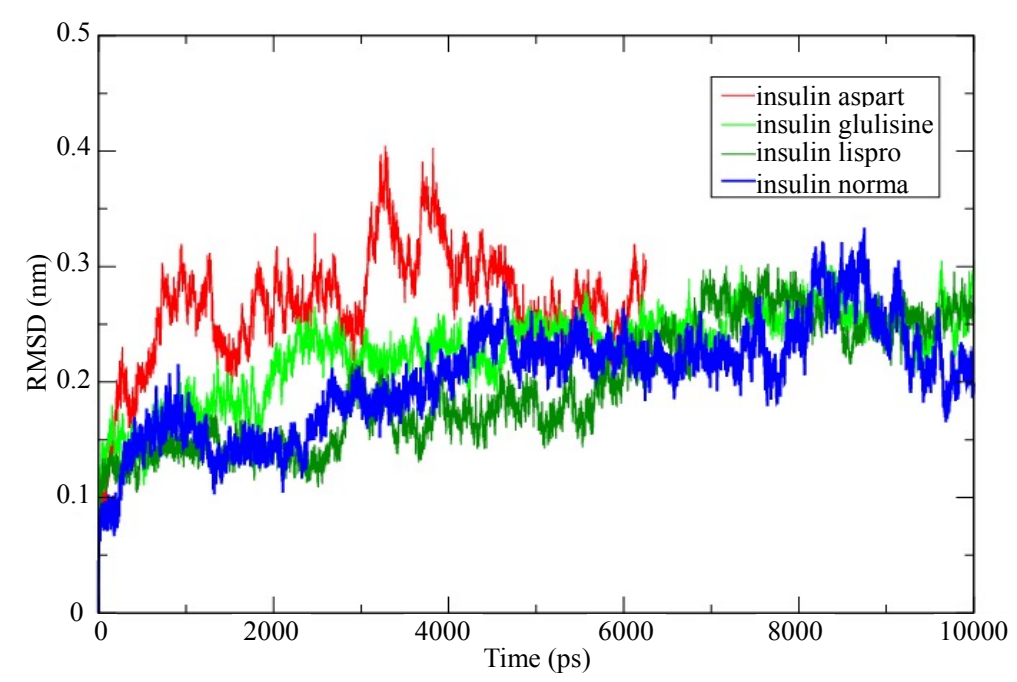

Figure 2. RMSD of backbone atoms (nm).

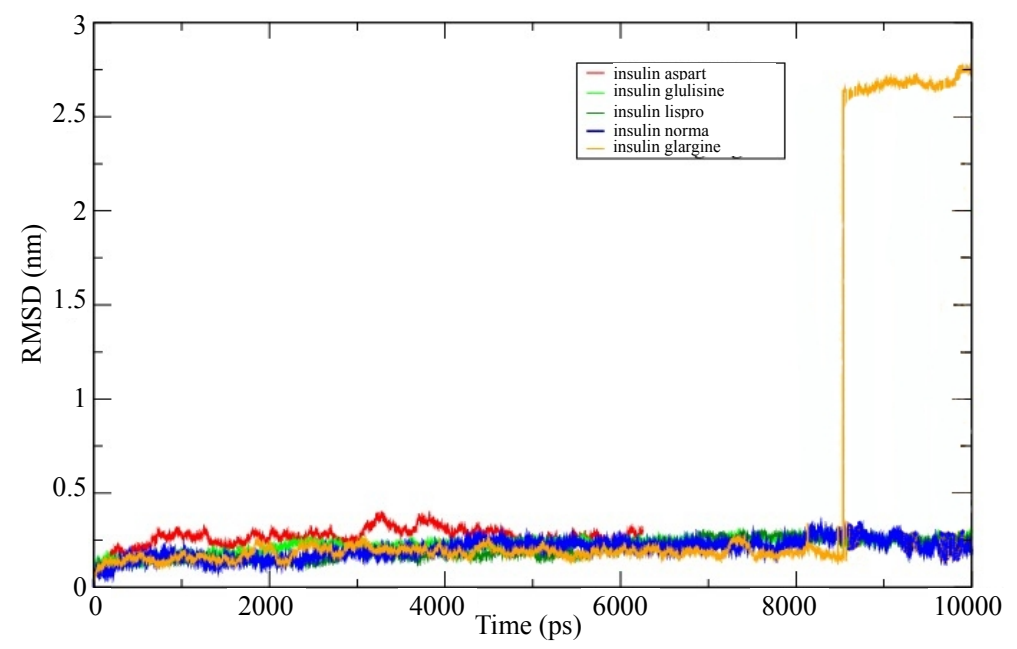

Figure 3. RMSD of backbone atoms (nm), including insulin glargine. 
These curves show that insulin glargine is the most flexible analogue. Its sharp shift at 8500 ps can be explained by existence of two positively charged arginine at the C-terminus of the B-chain and replacing the acidsensitive asparagine by glycine at the C-terminus of the A-chain.

Normal insulin has also several sharp shifts shown in Figure 4, however not so high.

\subsection{Root Mean Square Fluctuation Analysis}

The conformational mobility of atoms located in the residues was assessed by calculating the root mean square fluctuations (RMSF).

Figure 5 shows per-atom RMSF for all insulin ana- logues.

These curves demonstrate that insulin glargine is the most flexible analogue. Its curve can apparently be divided into two segments: one - for A-chain $(<308$ atom), another-for B-chain ( $>308$ atom). The previous MD studies of insulin have shown that A- and B-chains are differently flexible. To explain this phenomenon we suggest that their behaviour is different because of their mostly independent folding. Depending on the chosen diabetes treatment strategy, one may need rapid- or longacting insulin analogue. We suggest that to design the rapid-acting analogue, one should make substitutions in B-chain, while for achieving the long-lasting effect both chains should be modified.

The plot also shows that the normal insulin has RMSF

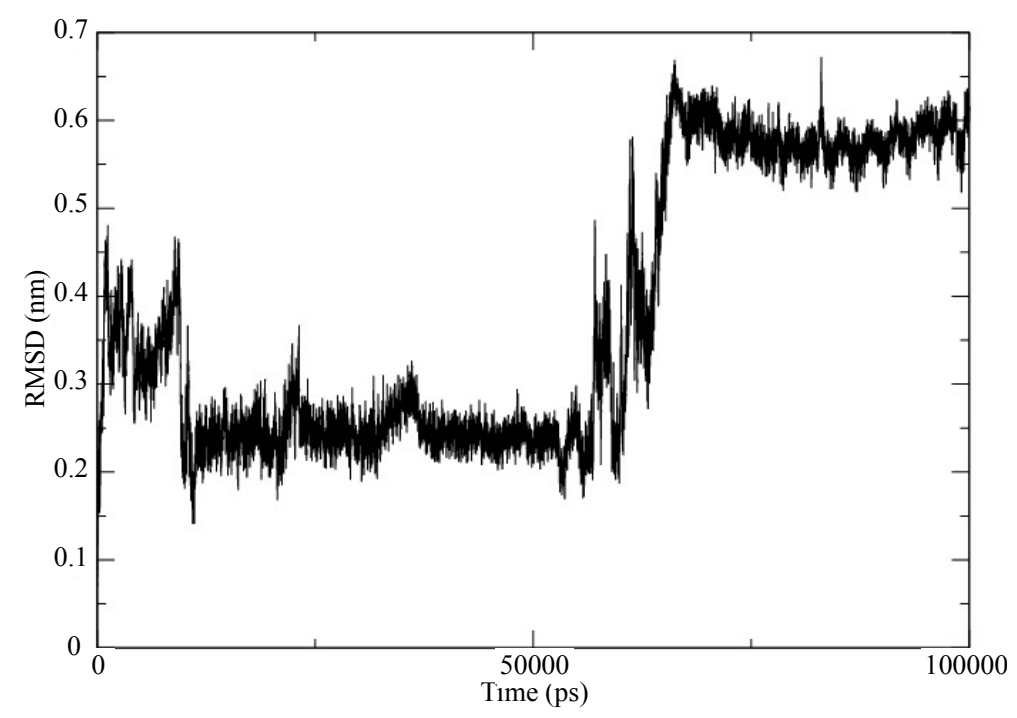

Figure 4. RMSD of backbone atoms (nm) for normal insulin (without substitutions) during $100,000 \mathrm{ps}$.

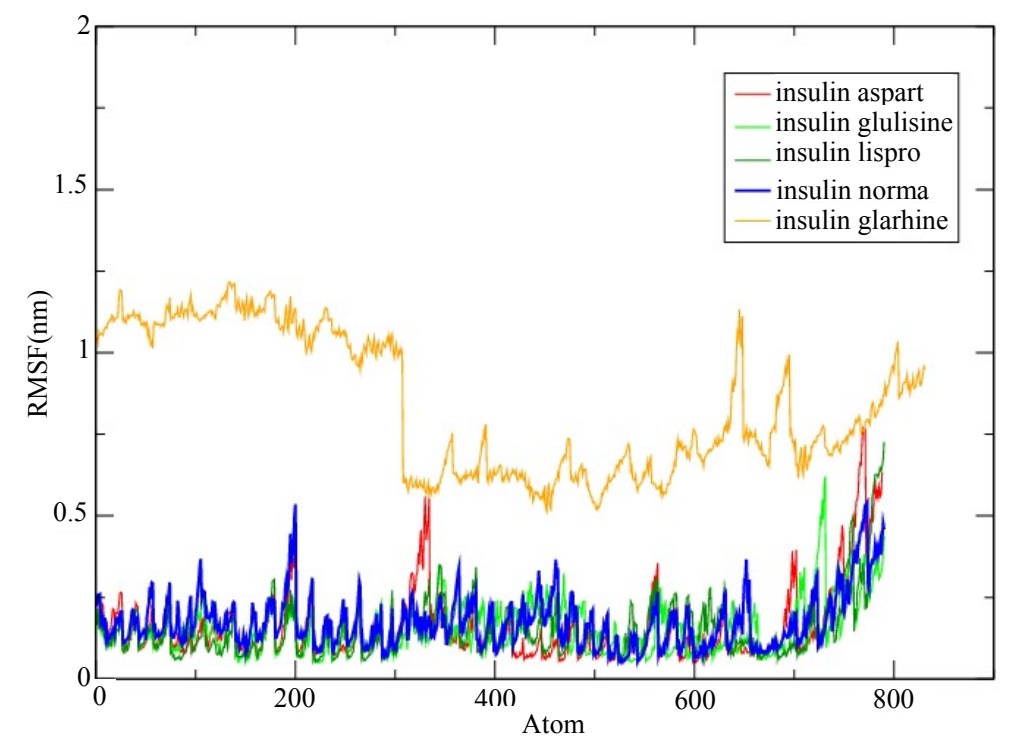

Figure 5. Per-atom RMSF (nm). 
at 100 atom $\left(\mathrm{Thr}^{\mathrm{A} 8}\right), 200$ atom $\left(\mathrm{Tyr}^{\mathrm{A} 8}\right)$ of A-chain and at 650 atom $\left(\mathrm{Arg}^{\mathrm{B} 22}\right)$ of $\mathrm{B}$-chain. The curve of insulin aspart has peaks at $\mathrm{N}$ - and C-termini (308 atom $\mathrm{Asn}^{\mathrm{A} 21} ; 750$ 788 atoms $-\mathrm{B} 28-\mathrm{B} 30)$, at 560 atom $\left(\mathrm{Tyr}^{\mathrm{B} 16}\right)$ and 700 atom $\left(\mathrm{Phe}^{\mathrm{B} 25}\right)$. The curve of insulin glulisine has a rather high peak at 720 atom $\left(\mathrm{Tyr}^{\mathrm{B} 26}\right)$. The least atom fluctuations has insulin lispro. There are slight fluctuations in the range: $540\left(\mathrm{Leu}^{\mathrm{B} 15}\right)-620\left(\mathrm{Glu}^{\mathrm{B} 21}\right)$, and at $\mathrm{C}$-terminus of B-chain.

Interestingly, the curves of normal insulin and insulin lispro have fluctuations only in alpha-helices and in the interhelices, while the curves of insulin aspart and insulin glulisine have fluctuations in the beta-strand. We have found that residues B16, B25 in insulin aspart and B26 in insulin glulisine are functionally important for the formation of dimers and are included in the classical site of the binding with IR.

Additionally, we performed the visual analysis of insulin analogues motion. The observations of high conformational flexibility of insulin aspart and insulin glargine, and compactness of insulin glulisine are in agreement with the above findings.

\section{DISCUSSION}

Since the discovery of insulin, a number of advances have been made to allow patients to mimic the normal pattern of insulin release more accurately and conveniently. However, insulin therapy needs to improve still further to allow near normalization of blood glucose with a low risk of hypoglycaemia and weight gain [26]. In this regard it is necessary to develop new pharmacologically effective insulin analogues with improved conformational flexibility necessary for manifestation of rapid and long-lasting effects.

Conformational flexibility analysis carried out in this work showed that insulin aspart has the highest mobility among rapid-acting insulin analogues. However, insulin glargine was the most flexible among all investigated substances. Interestingly, RMSF curve for insulin glargine can apparently be divided into two segments: onefor A-chain, another-for B-chain. To explain this phenomenon we suggested that their behaviour is different because of their mostly independent folding. This suggestion is in good agreement with previously obtained experimental data [27]. In addition, we showed that to design the rapid-acting analogue, one should make substitutions in B-chain, while for achieving the long-lasting effect, both chains should be modified. These results can be used for the design of new effective insulin-based drugs for the treatment of diabetes mellitus.

\section{ACKNOWLEDGEMENTS}

This study was supported by Saint Petersburg State University (projects
1.0.130.2010 and 0.37.141.2011).

\section{REFERENCES}

[1] Whiting, D.R., Guariguata, L., Weil, C. and Shaw, J. (2011) IDF diabetes atlas: global estimates of the prevalence of diabetes for 2011 and 2030. Diabetes Research and Clinical Practice, 94, 311-321. http://dx.doi.org/10.1016/j.diabres.2011.10.029

[2] Eisenbarth, G.S. (1986) Type I diabetes mellitus. A chronic autoimmune disease. The New England Journal of Medicine, 314, 1360-1368. http://dx.doi.org/10.1056/NEJM198605223142106

[3] Hua, Q., Hu, S., Jia, W., Wang, S. and Weiss, M.A. (2005) Toward the Active Conformation of Insulin. Stereospecific modulation of a structural switch in the B chain. The Journal of Biological Chemistry, 281, 24900-24909. http://dx.doi.org/10.1074/jbc.M602691200

[4] Olsen, H.B., Ludvigsen, S. and Kaarsholm, N.C. (1996) Solution structure of an engineered insulin monomer at neutral pH. Biochemistry, 35, 8836-8845. http://dx.doi.org/10.1021/bi960292+

[5] Kurtzhals, P. (2004) Engineering predictability and protraction in a basal insulin analogue: The pharmacology of insulin detemir. International Journal of Obesity, 28, 2328. http://dx.doi.org/10.1038/sj.ijo.0802746

[6] Bolli, G.B., Marchi, R.D. Di, Park, G.D., Pramming, S. and Koivisto, V.A. (1999) Insulin analogues and their potential in the management of diabetes mellitus. Diabetologia, 42, 1151-1167. http://dx.doi.org/10.1007/s001250051286

[7] Hermansen, K., et al. (2004) Insulin analogues (insulin detemir and insulin aspart) versus traditional human insulins (NPH insulin and regular human insulin) in basalbolus therapy for patients with type 1 diabetes. Diabetologia, 47, 622-629.

http://dx.doi.org/10.1007/s00125-004-1365-z

[8] Senesh, G., Bushi, D., Neta,A. and O. Yodfat (2010) Compatibility of insulin lispro, aspart, and glulisine with the Solo ${ }^{\mathrm{TM}}$ MicroPump, a novel miniature insulin pump. Journal of Diabetes Science and Technology, 4, 104-110.

[9] Swinnen, S.G.H.A., et al. (2009) Rationale, design, and baseline data of the insulin glargine (Lantus) versus insulin detemir (Levemir) treat-to-target (L2T3) study: A multinational, randomized noninferiority trial of basal insulin initiation in type 2 diabetes. Diabetes Technology and Therapeutics, 11, 739-743. http://dx.doi.org/10.1089/dia.2009.0044

[10] Chao, M., et al. (2010) Bioequivalence between two human insulin analogs in chinese population: Glulisine and lispro. Endocrine, 38, 48-52.

http://dx.doi.org/10.1007/s12020-010-9326-4

[11] Setter, S.M., Corbett, C.F., Campbell, R.K. and White, J.R. (2000) Insulin aspart: A new rapid-acting insulin analog. The Annals of Pharmacotherapy, 34, 1423-1431.

[12] Garg, S.K., Ellis, S.L. and Ulrich, H. (2005) Insulin glulisine: A new rapid-acting insulin analogue for the treatment of diabetes. Expert Opinion on Pharmacotherapy, 6, 
643-651. http://dx.doi.org/10.1517/14656566.6.4.643

[13] Bolli, G.B. and Owens, D.R. (2000) Insulin glargine. The Lancet, 356, 443-445.

http://dx.doi.org/10.1016/S0140-6736(00)02546-0

[14] Hansen, B.F., et al. (1996) Susteined signalling from the insulin receptor after stimulation with insulin analogues exhibiting increased mitogenic potency. Biochemical Journal, 315, 271-279.

[15] Hirsch, I.B. (2005) Drug Therapy Insulin Analogues. The New England Journal of Medicine, 352, 174-183. http://dx.doi.org/10.1056/NEJMra040832

[16] Karplus, M. and Petsko, G.A. (1990) Molecular dynamics simulations in biology. Nature, 347, 631-639. http://dx.doi.org/10.1038/347631a0

[17] Karplus, M. and McCammon, A. (2002) Molecular dynamics simulations of biomolecules. Nature Structural Biology, 9, 646-652. http://dx.doi.org/10.1038/nsb0902-646

[18] Budi, A., Legge, S., Treutlein, H. and Yarovsky, I. (2004) Effect of external stresses on protein conformation: A computer modelling study. European Biophysics Journal, 33, 121-129.

http://dx.doi.org/10.1007/s00249-003-0359-y

[19] Legge, F.S., Budi, A., Treutlein, H. and Yarovsky, I. (2006) Protein flexibility: Multiple molecular dynamics simulations of insulin chain B. Biophysical Chemistry, 119, 146-157. http://dx.doi.org/10.1016/j.bpc.2005.08.002

[20] Zoete, V., Meuwly, M. and Karplus, M. (2005) Study of the insulin dimerization: Binding free energy calculations and per-residue free energy decomposition. Proteins: Structure, Function, and Bioinformatics, 61, 79-93. http://dx.doi.org/10.1002/prot.20528
[21] Mark, A.E., Berendsen, H.J.C. and Gunsteren, W.F.V. (1991) Conformational flexibility of aqueous monomeric and dimeric insulin: a molecular dynamics study. Biochemistry, 30, 10866-10872. http://dx.doi.org/10.1021/bi00109a009

[22] Pronk, S., et al. (2013) GROMACS 4.5: A high-throughput and highly parallel open source molecular simulation toolkit. Bioinformatics, 29, 845-854. http://dx.doi.org/10.1093/bioinformatics/btt055

[23] Eswar, N., Eramian, D., Webb, B., Shen, M. and Sali, A. (2008) Protein structure modeling with MODELLER. Structural Proteomics. Methods in Molecular Biology, 426, 145-159. http://dx.doi.org/10.1007/978-1-60327-058-8_8

[24] Kaminski, G.A. and Friesner, R.A. (2001) Evaluation and reparametrization of the OPLS-AA force field for proteins via comparison with accurate quantum chemical calculations on peptides. The Journal of Physical Chemistry $B, \mathbf{1 0 5}, 6474-6487$. http://dx.doi.org/10.1021/jp003919d

[25] Humphrey, W., Dalke, A. and Schulten, K. (1996) VMD: Visual molecular dynamics. Journal of Molecular Graphics, 14, 33-38. http://dx.doi.org/10.1016/0263-7855(96)00018-5

[26] Heller, S., Kozlovski, P. and Kurtzhals, P. (2007) Insulin's $85^{\text {th }}$ anniversary-An enduring medical miracle. Diabetes Research and Clinical Practice, 78, 149-158. http://dx.doi.org/10.1016/i.diabres.2007.04.001

[27] Hua, Q. and Weiss, M.A. (2004) Mechanism of insulin fibrillation. The structure of insulin under amyloidogenic conditions resembles a protein-folding. The Journal of Biological Chemistry, 279, 21449-21460. http://dx.doi.org/10.1074/jbc.M314141200 